\title{
The Adolescent "Expanded Medical Home": School-Based Health Centers Partner with a Primary Care Clinic to Improve Population Health and Mitigate Social Determinants of Health
}

\author{
Margaret Riley, MD, Anna R. Laurie, MD, Melissa A. Plegue, MA, and \\ Caroline R. Richardson, $M D$
}

Introduction: Access to high-quality health care is a crucial social determinant of health. We describe the implementation of an "expanded medical home" partnering a primary care practice (the Ypsilanti Health Center [YHC]) with local school-based health centers (the Regional Alliance for Healthy Schools [RAHS]), and to assess whether this model improves access to and quality of care for shared patients.

Methods: Using the Consolidated Framework for Implementation Research, we define the steps in, barriers to, and facilitating factors in implementing the expanded medical home model. Visits and quality measures were assessed for patients seen by YHC only versus YHC/RAHS at baseline and during the intervention.

Results: At baseline, patients seen at YHC/RAHS had higher compliance with most quality metrics compared with those seen at YHC only. The proportion of shared patients significantly increased because of the intervention $(P<.001)$. Overall, patients seen in the expanded medical home had a higher likelihood of receiving quality metric services than patients in YHC only (odds ratio, 1.8; 95\% confidence interval, 1.57-2.05) across all measures.

Conclusions: Thoughtful and intentional implementation of an expanded medical home partnership between primary care physicians and school-based health centers increases the number of shared highrisk adolescent patients. Shared patients have improved compliance with quality measures, which may lead to long-term improved health equity. (J Am Board Fam Med 2016;29:339-347.)

Keywords: Access to Health Care; Adolescent Health; Community Medicine; Delivery of Health Care; Health Care Disparities; Medical Home; Patient-Centered Care; School Health Services; Social Determinants of Health

Adolescence is a critical stage in development in which health behaviors, including those that will last a lifetime, are adopted. ${ }^{1}$ These health behaviors are shaped by multiple social forces on personal, family, community, and national levels. ${ }^{2}$ These forces, also known as social determinants of health (SDOHs), are defined by the

\footnotetext{
This article was externally peer reviewed.

Submitted 14 September 2015; revised 1 February 2016; accepted 2 February 2016.

From the Department of Family Medicine, University of Michigan, Ann Arbor, MI.

Funding: none.

Conflict of interest: none declared.

Corresponding author: Margaret A. Riley, MD, Department of Family Medicine, University of Michigan, 1150 W Medical Center Dr, M7300 Medical Science I, SPC 5625, Ann Arbor, MI 48109-5625 (E-mail: marriley@med.umich.edu).
}

World Health Organization as "the conditions in which people are born, grow, live, work, and age," and are the influences that result in health inequities. ${ }^{3}$ HealthyPeople 2020, a national preventive health initiative in the United States, developed a framework to address 5 key areas of SDOHs. These notably include health and health care, a critical component of which is improving access to primary care. ${ }^{4}$ This is particularly applicable to the adolescent population who may experience numerous barriers to accessing health care, contributing to health inequities in this vulnerable population. ${ }^{5}$

The relationship between health outcomes and socioeconomic status (SES) has been well-researched; minority adolescents and those with low SES have been shown to have low rates of preven- 
tive care such as immunizations, poor management of common chronic diseases (eg, asthma and obesity), and limited access to mental health and substance abuse services. ${ }^{6-8}$ In addition, adolescents with low SES are less likely to have a source of primary care and yearly preventive visits. ${ }^{8,9}$ Adolescents with low SES, those who are uninsured, and those who are members of black/Hispanic families are less likely to receive health care in a medical home. ${ }^{6,10}$

School-based health centers (SBHCs) are an important point for access to health care by vulnerable adolescents. SBHCs overcome many barriers to care, such as cost to the patient, culture, age, time, and transportation. Adolescents who use SBHCs have been found to have increased rates of preventive visits and immunizations; improved chronic disease management outcomes for asthma, obesity, and mental health care; and decreased health care costs. ${ }^{6,11}$ Because of legitimate concerns around fragmentation of care for patients using SBHCs, the American Academy of Pediatrics endorses collaboration of community primary care providers
(PCPs) with SBHCs to reduce this risk, with the recommendation to provide special attention to facilitating communication between primary care sites and SBHCs. With new reimbursement models rewarding quality of care and improved health outcomes under the Affordable Care Act, collaboration between SBHCs and PCPs may be one way to simultaneously improve quality and reimbursement.

The Consolidated Framework for Implementation Research (CFIR) is a validated structure used to describe and define the constructs that influence the success of change implementation. ${ }^{12,13}$ Table 1 outlines the CFIR domains. Within our health system there has historically been little organized cooperation between PCPs and SBHCs. Using CFIR constructs to guide implementation, we sought to assess the process and impact of thoughtfully and intentionally partnering a large primary care clinic with local SBHCs in an "expanded medical home" model for adolescents with low SES. We hypothesized that implementing this expanded medical home partnership could help mitigate SDOHs

Table 1. Consolidated Framework for Implementation Research Constructs Describing Domains That Influence the Success of Implementing an Intervention

Domain Definition and Examples of Included Constructs

Intervention characteristics

Outer setting

Inner setting

Characteristics of individuals

Process
Describes the characteristics of the intervention being implemented into a particular organization; for example:

- if the intervention is developed internally or externally

- if the intervention can be adapted to meet local needs

- perceived complexity of the intervention

Consists of the economic, political, and social context outside of or encompassing the organization implementing change; for example:

- how well the organization understands and prioritizes patient needs, including barriers and facilitators to meet those needs

- external pressures on the organization, such as a need to meet external mandates, recommendations, and guidelines; pay-for-performance; and/or public or benchmark reporting

Includes the structural, political, and cultural contexts of the organization that may influence the implementation process; for example:

- the organization's capacity for change, which includes individuals' receptivity to the intervention and the extent that the organization rewards, expects, and supports the intervention

- the organization's readiness to change, which is affected by leadership engagement, resources available, and clear understanding of the intervention

Describes the characteristics of the individuals involved with the intervention and/or implementation process; for example:

- individuals' attitudes toward and value placed on the intervention

- individuals' perception of, relationship with, and commitment to the organization

Describes components of the process, from planning to evaluation; for example:

- the degree and quality of planning of the tasks needed to implement the intervention

- involvement of key individuals in the implementation, such as opinion leaders, formal leaders, champions, and change agents

Data from refs. 12 and 13. 
through improved access to and quality of primary care. This article describes how the intervention was implemented using the paradigms of relevant CFIR domains, and the impact of the expanded medical home on the quality of care for adolescent patient.

\section{Methods}

This implementation study received exempt status as quality improvement by the University of Michigan Internal Review Board (HUM00092711).

\section{Background Information on the Patients and Sites (CFIR Domains of Inner and Outer Settings)}

The University of Michigan Hospital and Health Systems (UMHS) Regional Alliance for Healthy Schools (RAHS) is a series of 6 SBHCs in middle and high schools serving youth aged 10-21 years. RAHS clinics are funded by a combination of revenue from insurance billing, support from UMHS, and grant funding, primarily from the Michigan Department of Health and Human Services. Services for uninsured patients and all services not covered by insurance (including co-pays) are free to patients in the age range. To qualify for grant funding for an RAHS clinic from the Michigan Department of Health and Human Services, $>50 \%$ of the students at the school must be eligible for free or reduced-fee lunch (family income $\leq 130 \%$ of the poverty level to qualify for free lunch, and $185 \%$ of the poverty level for reduced-fee lunch).

RAHS clinics are staffed by a full-time nurse practitioner, a full-time social worker, a half-time registered dietician, and a primary care physician (1 half day/week). The clinics provide comprehensive physical health services, including physical exams, immunizations, acute care, chronic disease management, and confidential care for minor consented services such as sexually transmitted infection testing and treatment; mental health counseling; and nutrition services. RAHS also provides assistance with meeting tangible needs such as housing and food by providing referrals to community resources, along with insurance enrollment. RAHS clinics are part of the "safety net" network in Michigan, and all students using the clinic are encouraged to have a PCP as well. Table 2 provides a summary of patient demographics and the services provided by RAHS.
The UMHS Ypsilanti Health Center (YHC) houses family medicine and pediatric clinics with 11 attending PCPs, 18 resident physicians, and additional physicians providing child psychiatry and developmental pediatrics services. Physicians at YHC are the most common PCPs listed by patients who use RAHS clinics. YHC and RAHS share the UMHS Epic electronic health record (EHR) and therefore can easily access and share patient information.

\section{Partnering YHC and RAHS: Bringing Individuals Involved Together (CFIR Domains of Characteristics of Individuals and Process)}

The idea of a pilot of the expanded medical home as a partnership to better care for shared high-risk patients was envisioned by RAHS and YHC leadership over a series of meetings identifying opportunities, barriers, and benefits for patients, providers, and the health centers. The model was then presented to RAHS and YHC providers and staff for feedback and to garner support and buy-in.

Table 2. Summary of Patient* Demographics and Services Provided by the Regional Alliance of Healthy Schools School-Based Clinics from July 2014 to June 2015

\begin{tabular}{|c|c|}
\hline At-risk youth seen (n) & 2200 \\
\hline Total visits (n) & 9338 \\
\hline \multicolumn{2}{|l|}{ Payer (\%) } \\
\hline Medicaid & 45 \\
\hline Uninsured & 30 \\
\hline Private insurance & 25 \\
\hline \multicolumn{2}{|l|}{ Sex $(\%)$} \\
\hline Male & 53 \\
\hline Female & 47 \\
\hline \multicolumn{2}{|l|}{ Race (\%) } \\
\hline Black & 47 \\
\hline White & 33 \\
\hline Unknown/unreported/refused to answer & 11 \\
\hline American Indian & 3 \\
\hline Asian & 3 \\
\hline Multiple Races & 3 \\
\hline Families assisted with insurance enrollment (n) & 407 \\
\hline $\begin{array}{l}\text { Families provided with assistance in meeting } \\
\text { tangible needs (food, housing, utilities, cash } \\
\text { assistance) (n) }\end{array}$ & 1500 \\
\hline $\begin{array}{l}\text { Youth receiving free vision services, including } \\
\text { glasses (n) }\end{array}$ & 355 \\
\hline Youth receiving free dental care (n) & 297 \\
\hline
\end{tabular}

Data are percentages unless otherwise indicated.

*The patients analyzed in this study are a subset of this group. 
A "meet and greet" over lunch at YHC was arranged to allow providers and staff from YHC and RAHS to connect, establish rapport, learn about services available at the different centers, and begin to develop trust.

\section{The Process of Implementation: Coordinating Care and Focusing on Quality Measures to Improve Population Health (CFIR Domain of Process)}

A letter signed by leadership from both sites was then sent to families of shared patients explaining that YHC is the patient's medical home and meant to be the primary source of care, but RAHS is an alternative if the patient cannot come to YHC, and that providers and staff will communicate and work together to coordinate care. An additional letter was sent to all RAHS patients explaining RAHS services, encouraging families to continue to connect with their PCP, and to become established with YHC if they do not yet have a PCP.

Providers from both sites worked to determine optimal means for communicating about shared patients (ie, a page sent to the PCP for urgent questions and a note routed through the EHR for routine communications) and to discuss ways that complementary care could be provided at the 2 sites (eg, a patient with an asthma exacerbation seen at YHC could get follow-up, spirometry, and reinforcement of the asthma action plan at RAHS). PCPs and RAHS providers committed to routinely routing notes to each other in the EHR for comanaged patients.

YHC had a "panel manager" who was tasked with running reports from the EHR to find shared patients who were missing recommended services including immunizations; asthma measures (asthma action plan, spirometry, and influenza vaccination); and well-child visits. For patients on these lists who were unable or unwilling to see their PCP, RAHS nurse practitioners would schedule them to be seen at the SBHC to close the gaps on any needed services.

\section{Measuring Improvements in Adolescent Quality Measures}

Quality of care was assessed by measuring compliance with recommended preventive health care measures (including yearly well-child visits and influenza vaccination rates) and chronic disease management measures for patients with asthma and/or obesity. The UMHS Quality Management Pro- gram determined compliance with these quality measures for patients seen at YHC alone (YHC only) versus shared care between YHC and RAHS (YHC/RAHS) at baseline (September 1, 2013, through August 31, 2014) and during the schoolyear intervention period (September 1, 2014, through June 30, 2015).

\section{Analysis}

To assess the quantity of shared patients, the proportion of patients who were seen at the expanded medical home (YHC/RAHS) was compared with all patients seen at YHC between baseline and during the intervention period using the Pearson $\chi^{2}$ test.

To assess compliance with quality measures (including well-child visits, influenza vaccination, nutrition and physical activity counseling for overweight and obese patients, and spirometry and asthma action plans for patients with asthma), the proportion of patients who received each of the recommended services was compared between those seen at YHC only and those seen in at YHC/ RAHS using the Pearson $\chi^{2}$ test or Fisher exact test, as appropriate. These comparisons were done separately at baseline and at the project's end for data collected during the school-year intervention.

To assess the specific impact of coordinating care within the expanded medical home, the change in quality measures across time was evaluated by comparing baseline and final time proportions separately within the YHC/RAHS and YHC-only groups using $\chi^{2}$ tests. These changes in proportions from baseline to follow-up were then compared between the 2 groups using a $z$-test to evaluate whether YHC/RAHS had significantly more improvement in quality measures than the YHC-only group. An overall measure of the effect that the expanded medical home had on the likelihood of receiving the recommended services was evaluated through a logistic regression, with a binary indicator for receiving the service as the outcome, and group (YHC/RAHS vs YHC only), time, and service type as covariates.

Qualitative data used for the analysis of the implementation process was derived from discussions at bimonthly staff meetings at both YHC and RAHS, communications between YHC and RAHS leadership, and reports from patients who had interactions with PCPs and RAHS during the expanded medical home intervention year. Themes 
that recurred in multiple communications and/or meeting notes were identified by the project leads (MR and CRR) and classified according to the CFIR framework. Numbers of shared patients during the intervention year were monitored and used as a marker of the success of the implementation process.

\section{Results}

\section{Changes Over the Intervention School Year: Shared Patients}

In the 10-month school-year intervention period, the number of YHC/RAHS shared patients increased from an average of 16 seen per month at baseline to 24 per month during the intervention. This reflects a statistically significant increase in the proportion of shared patients since the implementation of the expanded medical home: shared patients increased from 13\% (192 of $1471)$ at baseline to $19 \%$ (240 of 1275) at follow-up ( $P$ value $<.001)$.

\section{Assessing Changes in Quality Measures}

At baseline, patients seen at YHC/RAHS had higher compliance with most quality metrics compared with those seen at YHC only, though this difference met statistical significance for only 2 measures: documented nutrition and physical activity counseling for patients with higher body mass index $(P<.001)$ and influenza vaccination $(P=.018)$ (Table 3).

For the majority of the quality measures, both YHC/RAHS and YHC only showed improvement over the course of the intervention year, though few were statistically significant. Well-child visits at YHC only $(65.5 \%$ vs $69.9 \% ; P=.03)$ and asthma action plans for both groups $(65.0 \%$ vs $92.3 \%$ in YHC/RAHS $[P=.02]$ and $46.0 \%$ vs $77.2 \%$ in YHC only $[P<.001])$ were significantly higher during the intervention year when compared with baseline.

\section{Assessing Changes in Shared Patients Compared with YHC-Only Patients at Baseline versus During the Intervention}

A comparison of services received at baseline with those received during the expanded medical home intervention period showed notable differences between shared patients versus YHC-only patients. Overall, patients seen in the expanded medical home had a higher likelihood of receiving quality metric services than patients seen at YHC only (odds ratio, 1.8; 95\% confidence interval, 1.572.05) across all measures.

When considering individual quality metrics, there were no statistically significant differences between the improvements in the 2 groups (ie, the difference in between-group differences), likely because of the small sample size for each individual metric. However, there were notable trends toward widening the gap in the improvement in quality measures met for YHC/RAHS patients compared with those seen at YHC only. The rates of patients who had a well-child visit in the expanded medical home compared with YHC only was statistically different during the intervention period $(P=.03)$, though not in the baseline period $(P=.14)$. The difference in patients meeting asthma quality measures in the expanded medical home compared with YHC only was also notably different at baseline than during the intervention (spirometry difference: $P=.98$ at baseline, $P=.09$ during intervention; influenza vaccine for asthma patients difference: $P=.89$ at baseline, $P=.09$ during intervention; asthma action plan difference: $P=.19$ at baseline, $P=.10$ during intervention) (Figure 1).

\section{Factors Facilitating and Barriers to Implementation Success}

Both the inner and outer contexts helped to enable the success of implementing this model. YHC sees high-risk patients with a low SES who have a significant need for and traditionally low uptake of health services. This high patient need facilitated the expanded medical home as a patient-centered approach to providing health care services to patients within the community. A growing shift in the larger UMHS health system away from the traditional fee-for-service model toward paying for quality measures provided an additional external force to motivate PCPs to focus on creative strategies to improve population health within the practice at YHC.

Some individuals involved in the partnership facilitated the implementation, whereas others were barriers. The medical directors from YHC and RAHS, in addition to many PCPs and SBHC providers, were very supportive of partnering and met regularly to review challenges and successes within the implementation process. However, some PCPs expressed resistance at staff meetings 


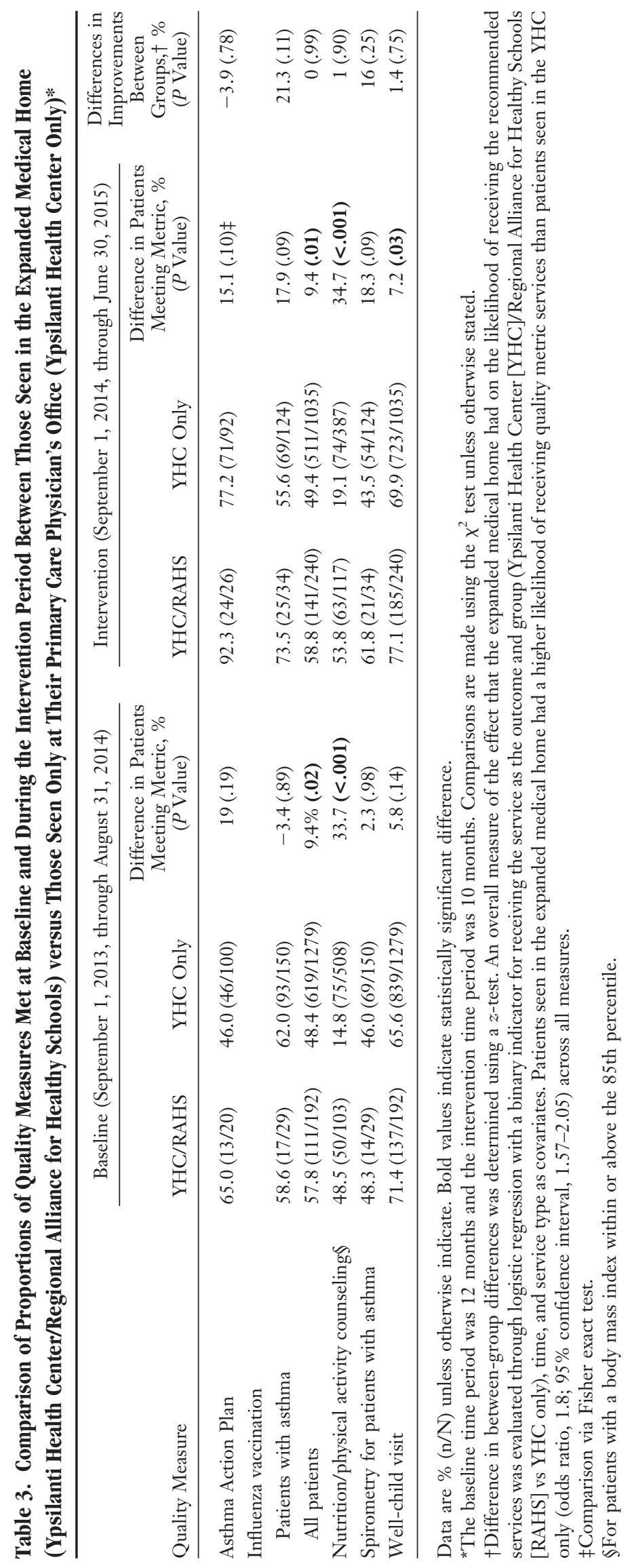


Figure 1. Comparison of the percentage of quality measures met at baseline and at the end of the intervention period for patients in the expanded medical home (Ypsilanti Health Center [YHC]/Regional Alliance for Healthy Schools [RAHS]) versus those seen only at their primary care physician's office (YHC only).

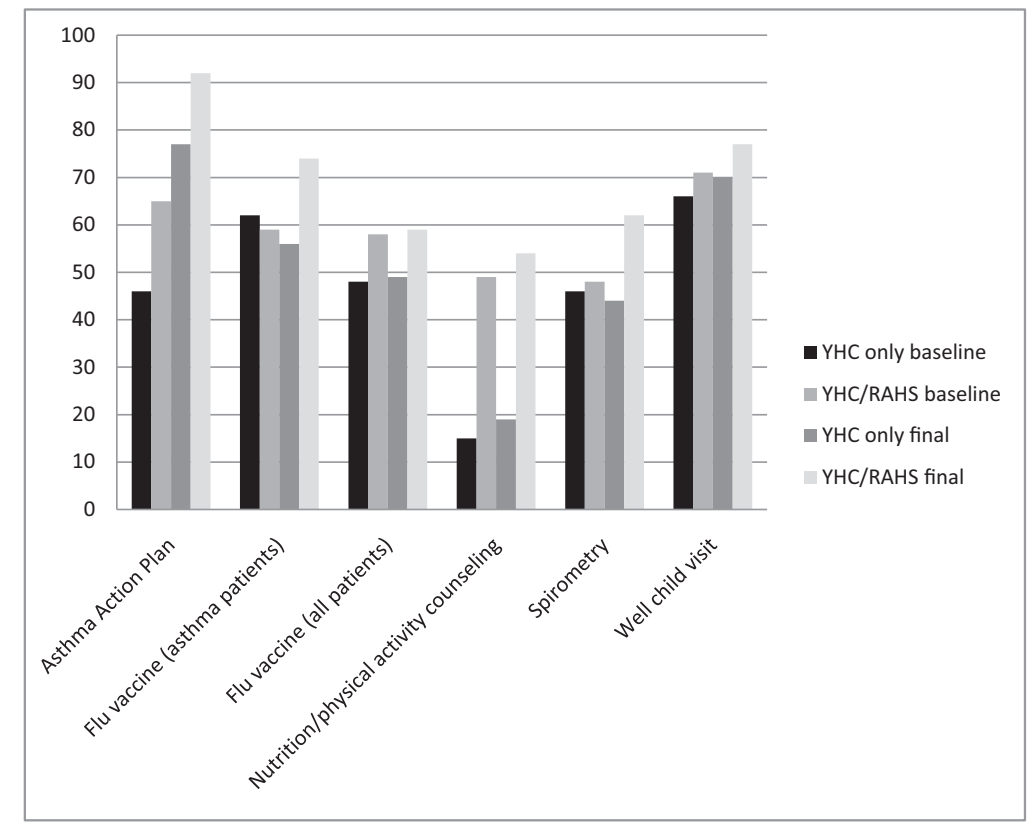

and via E-mail to the idea of intentionally sharing patients, primarily because of a sense of loss of ownership and erosion of the physician-patient relationship; some patients reported to RAHS staff that their PCP specifically advised against receiving any services at RAHS. As a result, the SBHC staff related at staff meetings a hesitancy to communicate with certain PCPs for fear of disrespect or retribution, and some patients became confused about the legitimacy of the partnership.

The process of implementation was carefully planned and mapped out, contributing to building a successful expanded medical home model. Teams from the SBHC and PCPs were engaged in the planning process, champions from both groups emerged and facilitated the model, and leadership spoke out clearly and frequently in favor of the partnership. Some implementation difficulties occurred with unforeseen barriers to data collection, which limited partnership capabilities and wasted time for both groups. For example, gap reports for needed services were difficult to run and often had errors, and there were limitations on data that could be pulled from the EHR (eg, human papillomavirus vaccination rates were inaccessible).

\section{Discussion}

We found that the adolescents with low SES in this study seen both at an SBHC and by a PCP had better adherence with preventive and chronic disease management quality measures at baseline than those seen by a PCP alone. This is consistent with previous research that showed that SBHC users attend more well-child visits, have increased immunization rates, and have better compliance with asthma measures. ${ }^{14,15}$ This study provides novel information about the power of care coordination between PCPs and SBHCs, and guidance on how to effectively implement partnership. We found that when the PCPs and local SBHCs partnered in an "expanded medical home," significantly more patients were shared between both sites, and more patients benefited from access to care within the expansion of their medical home. In addition, when looking across all measures, patients seen in the expanded medical home had a higher likelihood of receiving quality metric services than patients seen only in a PCP's office.

Using the CFIR framework to guide, analyze, and describe implementation provided us with a foundation to understand the multitude of factors that influence the success of implementing a new model. The CFIR vocabulary and constructs al- 
lowed us to identify clearly facilitating factors and barriers among the patient population, clinical settings, the larger health system, and the planning process.

This expanded medical home benefited adolescent patients and their parents, PCPs, and SBHCs (the CFIR inner and outer settings). Shared patients had increased frequency of well-child visits and associated preventive services, in addition to improved completeness of care for asthma and obesity. When appropriate, follow-up after PCP encounters were coordinated to occur at the SBHC, decreasing time away from school and averting a potential loss of parental income to attend multiple visits. PCPs benefitted from patient panels with improved adherence to recommended services, leading to increased reimbursement for better quality of care. PCPs also gained new patients as the SBHC shunted patients without an established PCP to their expanded medical home partner office. Finally, SBHCs benefitted from increased patient visits resulting from PCP referrals, increased legitimacy with patients and families because of the connection with the PCP, and improved institutional support through linkage with the PCP's office. This model also improved relationships between SBHC staff and PCPs, leading to better communication, better coordination, and potential decreases in redundant care.

There are a number of limitations to this study. It was a pilot of an expanded medical home between 1 large primary care office and 6 SBHCs, and may not be generalizable to other health systems and settings. We had significant issues with data management throughout the study, from difficulty running accurate reports of gaps in needed services for patients to limitations in the quality measures that the Quality Management Program was able to pull. Desired quality measures such as human papillomavirus vaccination and chlamydia screening were not available at the time of the study.

Because of the nature of the data there were additional analysis limitations. This is an ecological study, where the effects of risk-modifying factors on health or other outcomes based on populations are defined either geographically or temporally. (Both risk-modifying factors and outcomes are averaged for the populations in each geographical or temporal unit and then compared using standard statistical methods.) Because only aggregate data were available, we were unable to incorporate individual heterogeneity over time and to each quality measure. In particular, this led to the assumption of independence of the quality measures received by the same individual across years. However, because these quality measures are reflective of procedures that should be performed annually, such an assumption does not seem unreasonable. Analyses were also limited by the small sample size for some of the individual quality measures.

\section{Conclusion}

Unmet health care needs are a significant SDOH for adolescents and can lead to adverse health outcomes both immediately and in adulthood. Access issues are one of the primary reasons adolescents do not receive adequate health care. ${ }^{5,16,17}$ By thoughtfully and intentionally expanding the idea of what makes a medical home to partner PCPs with SB$\mathrm{HCs}$, these access issues can be significantly mitigated for youth with low SES. In addition to providing data on how care quality is improved within an expanded medical home, this study can help to inform best practices for implementing these partnerships. Further research is needed to assess additional short-term benefits in patient care quality and long-term effects on health outcomes for youth seen within an expanded medical home.

\section{References}

1. Sawyer SM, Afifi RA, Bearinger LH, et al. Adolescence: a foundation for future health. Lancet 2012; 379:1630-40.

2. Viner RM, Ozer EM, Denny S, et al. Adolescence and the social determinants of health. Lancet 2012; 379:1641-52.

3. World Health Organization, Commission on Social Determinants of Health. Closing the gap in a generation: health equity through action on the social determinants of health. Available from: http:// www.who.int/social_determinants/thecommission/ finalreport/en/. Accessed April 1, 2016

4. Office of Disease Prevention and Health Promotion. HealthyPeople 2020. Social determinants of health. Washington, DC: US Department of Health and Human Services. Available from: http://www.healthypeople.gov/2020/topics-objectives/topic/social-determinants-health. Accessed March 24, 2016.

5. Hargreaves DS, Elliott MN, Viner RM, Richmond TK, Schuster MA. Unmet health care need in US adolescents and adult health outcomes. Pediatrics 2015;136:513-20. 
6. Keeton V, Soleimanpour S, Brindis CD. Schoolbased health centers in an era of health care reform: building on history. Curr Probl Pediatr Adolesc Health Care 2012;42:132-56.

7. Goodman E. The role of socioeconomic status gradients in explaining differences in US adolescents' health. Am J Public Health 1999;89:1522-8.

8. Newacheck PW, Hung YY, Park MJ, Brindis CD, Irwin CE Jr. Disparities in adolescent health and health care: does socioeconomic status matter? Health Serv Res 2003;38:1235-52.

9. Irwin CE, Adams SH, Park MJ, Newacheck PW. Preventive care for adolescents: few get visits and fewer get services. Pediatrics 2009;123:e565-72.

10. Adams SH, Newacheck PW, Park MJ, Brindis CD, Irwin CE. Medical home for adolescents: low attainment rates for those with mental health problems and other vulnerable groups. Acad Pediatr 2013;13: 113-21.

11. Council on School Health. School-based health centers and pediatric practice. Pediatrics 2012; 129:387-93.
12. Consolidated Framework for Implementation Research (CFIR) [homepage on the Internet]. Available from: http://cfirguide.org/index.html. Accessed December 2015.

13. Damschroder LJ, Aron DC, Keith RE, Kirsh SR, Alexander JA, Lowery JC. Fostering implementation of health services research findings into practice: a consolidated framework for advancing implementation science. Implement Sci 2009;4:50.

14. Allison MA1, Crane LA, Beaty BL, Davidson AJ, Melinkovich P, Kempe A. School-based health centers: improving access and quality of care for lowincome adolescents. Pediatrics 2007;120:e887-94.

15. Webber MP, Carpiniello KE, Oruwariye T, Lo Y, Burton WB, Appel DK. Burden of asthma in innercity elementary schoolchildren: do school-based health centers make a difference? Arch Pediatr Adolesc Med 2003;157:125-9.

16. Ford CA, Bearman PS, Moody J. Foregone health care among adolescents. JAMA 1999;282:2227-34.

17. National Research Council, Institute of Medicine. Adolescent health services: missing opportunities. Washington, DC: National Academies Press; 2009. 